\title{
Chirality transfer from Graphene Quantum Dots
}

\author{
M. Vázquez-Nakagawa, ${ }^{a}$ L. Rodríguez-Pérez, ${ }^{a}$ M. A. Herranz ${ }^{* a}$ and N. Martín ${ }^{* a, b}$
}

\begin{abstract}
Chiral graphene quantum dots were prepared by acidic exfoliation and oxidation of graphite, dialysis, and esterification with enantiomerically pure $(R)$ or $(S)$-2-phenyl-1-propanol. Circular dichroism studies support the formation of supramolecular aggregates with pyrene molecules, where a transfer of chirality occurs from the chiral graphene quantum dots to the pyrene.
\end{abstract}

Carbon is one of the most abundant elements in nature, which has the singular feature of forming different allotropes and nanostructured forms. ${ }^{1}$ In particular, fullerenes, ${ }^{2}$ carbon nanotubes ${ }^{3}$ and graphene ${ }^{4}$ have provoked a great excitement in different research fields due to their remarkable properties and potential applications in materials science, optoelectronics, photovoltaics or biomedical sciences. ${ }^{5}$ However, large scale chemically pure forms of fullerenes and carbon nanotubes are still not easily affordable and are economically costly. In contrast, graphite is an inexpensive starting material for the production of graphene or graphene quantum dots (GQDs) considering a broad range of chemical and physical methods. ${ }^{6}$

Besides a simple fabrication method and a low production cost, GQDs have received considerable attention because of their electronic and optical properties, fine biocompatibility and low toxicity, which hold great promise for applications such as bioimaging, medical diagnosis, photovoltaic devices and catalysis. ${ }^{7}$ Nevertheless, when considering the potential applications of GQDs, chirality is an important aspect that can severely influence the performance and that has not been addressed so far. Natural processes have many examples where chiral compounds have a major role in molecular recognition, chemistry, biology and medicine, and an understanding of the fundamental concepts relevant to chirality in nanostructures is important for the further advancement of nanoscience and nanotechnology. ${ }^{8}$

Chiral quantum dots ( $\mathrm{CdS}, \mathrm{CdSe}, \mathrm{CdTe}, \mathrm{ZnS}$ ) have been prepared by a fast microwave-induced heating of the corresponding precursors in the presence of enantiomerically pure stabilizing ligands or via the conventional hot injection technique followed by a phase transfer in the presence of an appropriate chiral stabilizer. In the examples reported to date, the chiral stabilizing ligands and the induced chirality effects have a crucial role in the optical properties, in particular in luminescence sensing and chiral recognition of enantiomers. ${ }^{9}$

In the case of carbon nanostructures, chirality has been barely explored. Our research group has reported the highly efficient synthesis of enantiomerically pure derivatives of fullerene and endohedral fullerenes with total control of the stereochemical outcome using metallic catalysis and/or organocatalysts under very mild conditions. ${ }^{10} \mathrm{~A}$ further degree of complexity arises with carbon nanotubes, since each nanotube chirality is produced as an enantiomeric pair of nanotubes with opposite helicity. Research efforts in the recognition and separation of chiral carbon nanotubes have been based in the diameter selectivity with a variety of systems or in utilizing chiral ditopic receptors. $^{5 b, 11}$

Here, for a first time to the best of our knowledge, we proof the principle that chiral graphene quantum dots (CGQDs) can be obtained by reaction of oxidized GQDs with enantiomerically pure $(R)$ or $(S)$-2-phenyl-1-propanol and that their chirality can be efficiently transferred to the supramolecular assemblies formed with small molecules such as pyrene.

GQDs were obtained following a similar procedure to the one recently reported by Haino et al., ${ }^{12}$ which consists in the exfoliation and oxidative cutting of graphite in a mixture of concentrated $\mathrm{H}_{2} \mathrm{SO}_{4}$ and $\mathrm{HNO}_{3}(3 / 1 \mathrm{v} / \mathrm{v})$ at 120 oc for $48 \mathrm{~h}$, followed by neutralization of the excess of acid and a dialysis process. Initial transmission electron microscopy measurements (TEM) of the GQDs showed the presence of isolated GQDs together with bigger objects, as a consequence of the strong tendency to aggregation of GQDs due to the face-to-face attraction between them (Fig. 1). In order to obtain a more homogeneous size distribution, the as-prepared water soluble GQDs were separated with a Sephadex G-25 gel column into two fractions. ${ }^{13}$ The second and more abundant fraction yields a distributio

\footnotetext{
a. Departamento de Química Orgánica I, Facultad de Química, Universidad Complutense de Madrid, Avda. Complutense s/n, 28040 Madrid, Spain.

${ }^{b .}$ E-mail: maherran@ucm.es, nazmar@ucm.es

c. IMDEA-Nanociencia, c/Faraday 9, Campus Cantoblanco, 28049 Madrid, Spain

† Electronic Supplementary Information (ESI) available: See DOI: 10.1039/x0xx00000x
} 
of $\mathrm{GQD}_{\mathrm{s}}$ with a mean value of $22 \pm 4 \mathrm{~nm}$ based on TEM analysis, although most of the GQDs are, in fact, multiple layered (Fig. S1, ESI).
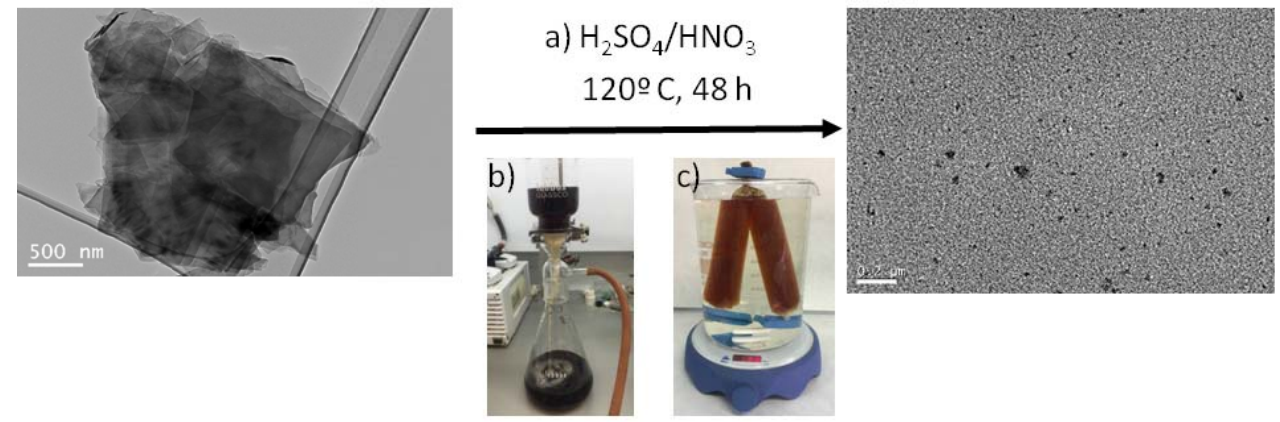

Fig. 1 Synthesis of GQDs starting from graphite and following a three step process: a) oxidation with mineral acids, b) neutralization and filtration and, c) dialysis. Scale bars = $500 \mathrm{~nm}$ (Graphite), $200 \mathrm{~nm}$ (GQDs).

The crystalline structure of the GQDs was investigated by X-ray diffraction (XRD) and the pattern features broad signals, where at $2 \theta=26.7(0.338 \mathrm{~nm})$ is observed the $(002)$ interlayer spacing of graphite, which is consistent with the preparation method used (Fig. S2, ESI). ${ }^{7}$

In the UV-vis absorption spectrum of the GQDs are observed the peaks at $\sim 230 \mathrm{~nm}$ due to the $\pi$ - $\pi^{*}$ transition of aromatic $\mathrm{C}=\mathrm{C}$ bonds and a band at $\sim 260 \mathrm{~nm}$ related with the $\mathrm{n}-\pi^{*}$ transition of $\mathrm{C}=\mathrm{O}$ bonds. ${ }^{14}$ Upon excitation with a $\lambda_{\text {exc }}=350 \mathrm{~nm}$, the as-prepared GQDs display a strong emission at $\sim 520 \mathrm{~nm}$ responsible of the light yellow color visible to the naked eye (Fig. 2).

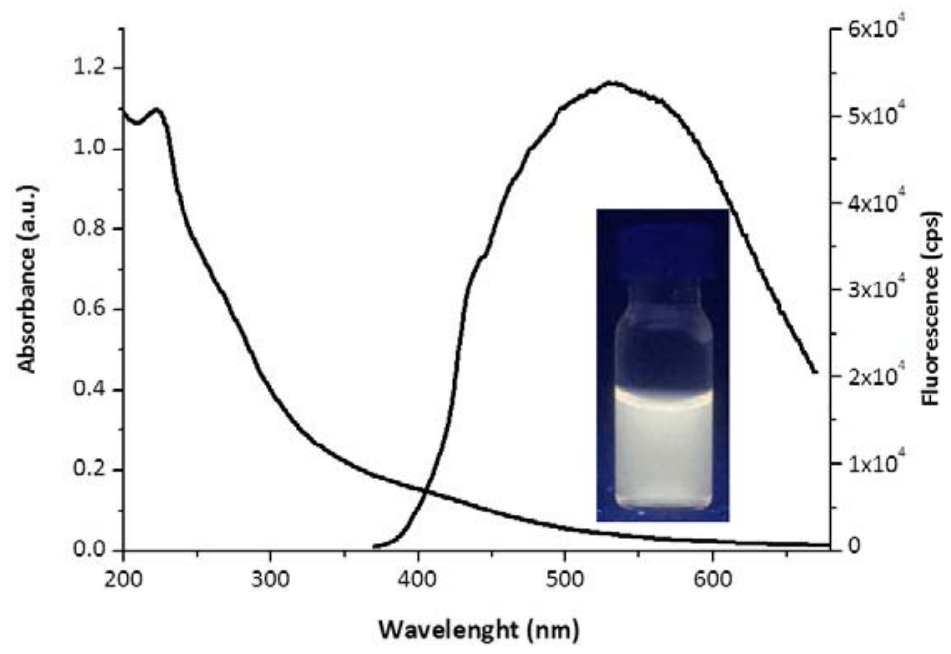

Fig. 2 UV-vis absorption and fluorescence spectra $\left(\lambda_{\text {exc }}=350 \mathrm{~nm}\right)$ of GQDs in $\mathrm{H}_{2} \mathrm{O}$. Inset: photograph of the GQD aqueous solution taken under $365 \mathrm{~nm}$ UV light.

GQDs were further treated with thionyl chloride under heating conditions to convert the carboxylic acids at the edges to acid chlorides that where reacted in situ with $(R)$ or $(S)$-2-phenyl-1-propanol to form enantiomerically pure esters (E), which yielded chiral graphene quantum dots (CGQDs) (Fig. 3). For synthetic details, see the ESI. 

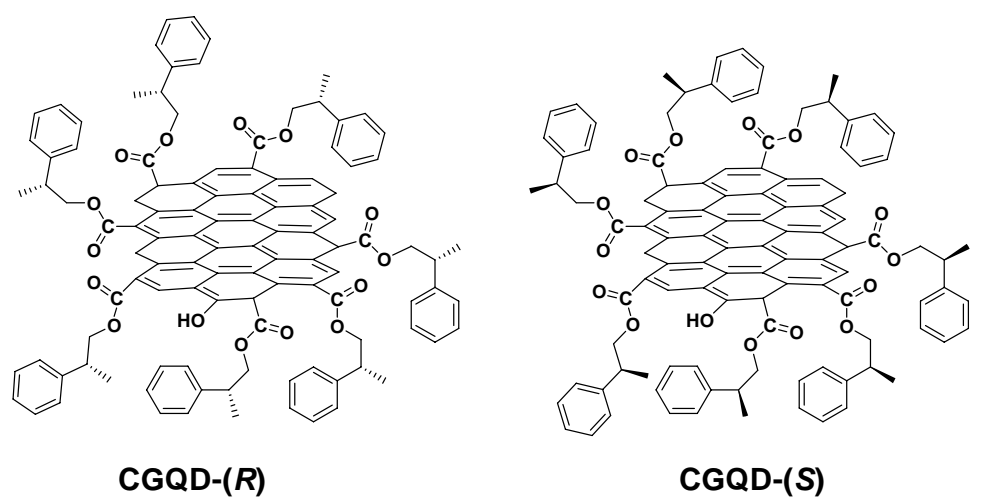

Fig. 3 Chiral graphene quantum dots obtained from esterification reaction with enantiomerically pure alcohols.

The first evidence of the successful covalent functionalization of GQDs was obtained from TGA under inert atmosphere. Fig. 4 shows the thermogravimetric profiles of graphite, GQDs and the CGQDs. Graphite remains stable in the experimental conditions while at 600 으 a weight loss of around $37 \%$ is observed for GQDs, which could be assigned to the removal of stable carboxylic acid groups at the edges of the GQDs structure. For CGQDs, an additional weight loss of $9 \%$ is observed at this temperature, which can be attributed to the decomposition of the ester moieties. The GQDs skeleton decomposes between 700-900 ㅇ (Fig. 4 and Fig. S3).

Further support on the covalent anchoring of the functional groups to the GQDs was obtained from NMR. In the ${ }^{13} \mathrm{C}$ NMR spectra of CGQDs additional signals to those of the GQDs are observed in the range of 120-150 ppm due to the presence of the phenyl substituents and the remaining carboxylic acids $(\sim 180 \mathrm{ppm})$ are distinguished from the formed esters $(\sim 168 \mathrm{ppm})$. In addition, the carbons of the methylene, methyne and methyl groups are observed at $67.4,41.4$ and $17.3 \mathrm{ppm}$, respectively (Fig. S4).

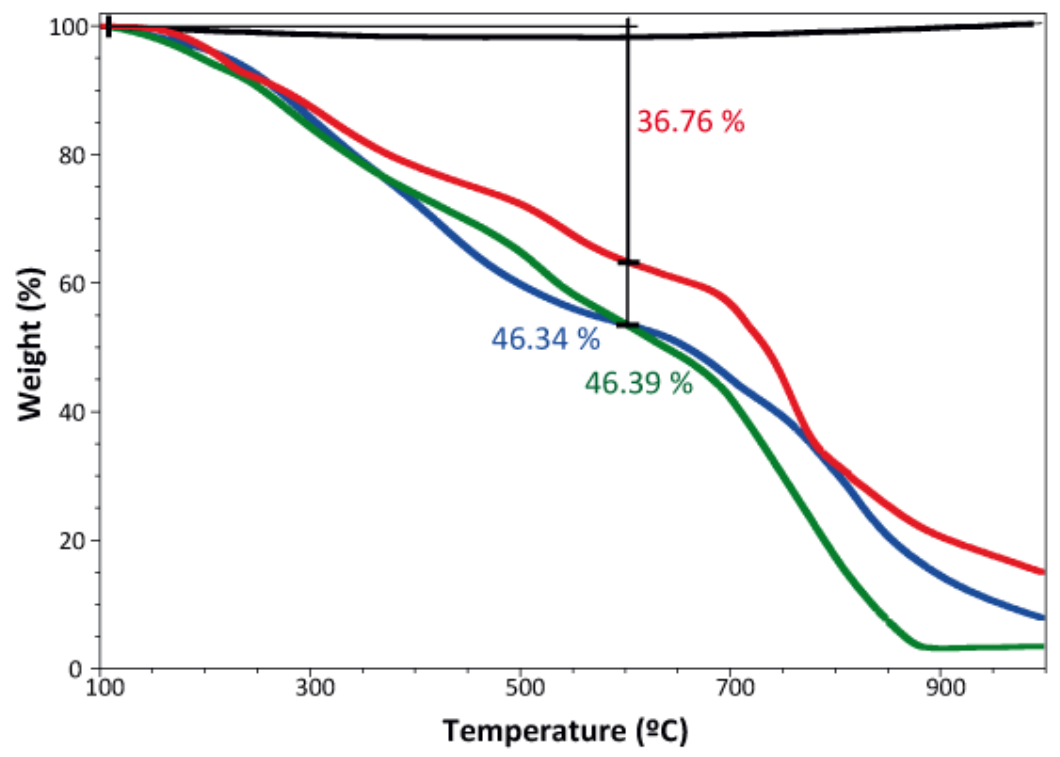

Fig. 4 TGA analyses under inert atmosphere of graphite (black), GQDs (red), CGQDs-(R) (blue) and CGQDs-(S) (green).

The FTIR spectra of CGQDs showed clear stretching vibrations of $C=O$ at $1731-1727 \mathrm{~cm}^{-1}$ combined with the asymmetric and symmetric stretching vibrations of C-O-C (around $1300 \mathrm{~cm}^{-1}$ and $1200 \mathrm{~cm}^{-1}$ ) in the ester groups. In addition, the band due to the skeletal in-plane vibration of $\mathrm{C}=\mathrm{C}$ is observed at $1592 \mathrm{~cm}^{-1}$ for GQDs and CGQDs (Fig. 5).

In Raman measurements, the characteristic $D$ and $G$ bands of GQDs at around $1387 \mathrm{~cm}^{-1}$ and $1590 \mathrm{~cm}^{-1}$, respectively, are observed for all samples (see Fig. S5 in ESI). No appreciable differences are observed between GQDs and CGQDs in the Raman spectra. The $I_{D} / I_{G}$ values range from 0.88-0.93, which point to partially disordered crystal structures, arising from the small $\mathrm{sp}^{2}$ cluster size. $^{15}$ 


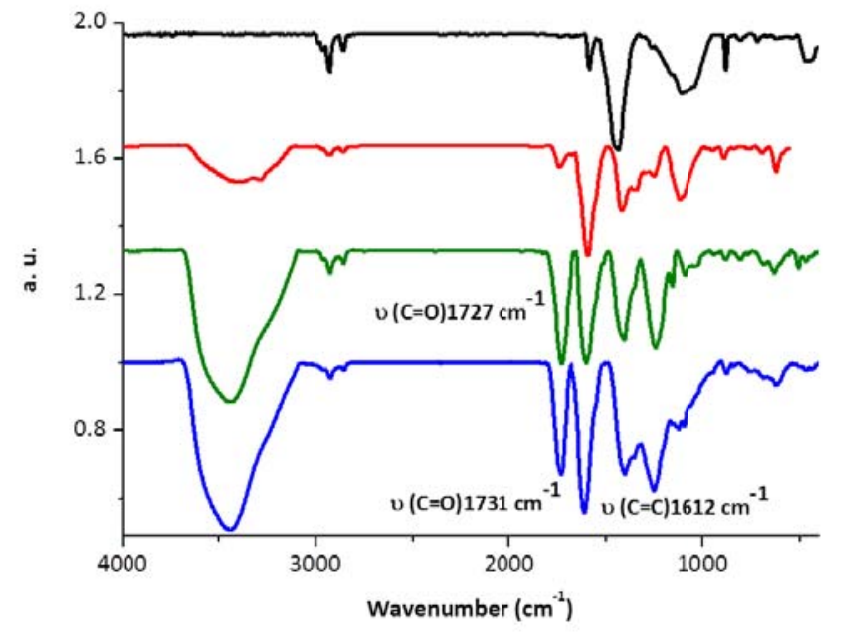

Fig. 5 FTIR spectra of graphite (black), GQDs (red), CGQDs-(R) (blue) and CGQDs-(S) (green).

The XRD patterns of both CGQDs were quite similar and comparable to that of the as-prepared GQDs (Fig. S2), displaying broad (002) peaks around 25 $(0.356 \mathrm{~nm})$, which were very close to the graphite (002) lattice spacing. ${ }^{7}$

In the TEM images of CGQDs (Fig. S6), dark spots with different sizes can be clearly seen. The small spots have diameters between 3-5 nm, although there is a prevalent population of large dark spots composed of several GQDs. Atomic force microscopy (AFM) measurements showed the presence of spherical morphologies where many agglomerates are present, the small benzylic groups introduced in the CGQDs do not interfere with the selfaggregation of GQDs (Fig. S7).

The UV-vis absorption bands of CGQDs in $\mathrm{H}_{2} \mathrm{O}$ appear at 225-300 $\mathrm{nm}$ and represent the typical absorption of an aromatic $\pi$ system, similar to the as-prepared GQDs (Fig. S8). The obtained CGQDs are strongly photoluminescent, upon excitation at $350 \mathrm{~nm}$ exhibited emission maxima at $\sim 530-570 \mathrm{~nm}$, which are slightly red-shifted when compared with the as-prepared GQDs (Fig. 2 and Fig. S7).

The chiroptical properties of the CGQDs where investigated by circular dichroism (CD) in $N$-methylpirrolidone (NMP). Since the functional groups introduced in the GQDs skeleton do not absorb over $300 \mathrm{~nm}$, pyrene was added to the solution, which absorbs strongly in the UV-vis region and is able to interact strongly with the GQDs by $\pi-\pi$ stacking forces as previously demonstrated with other carbon nanoforms. ${ }^{16}$ As expected, pyrene itself does not show a dichroic signal, but in the presence of the chiral material (GQD-E(R) or GQD-E(S)) provides a clear CD response (Fig. 6 and Fig. S9).

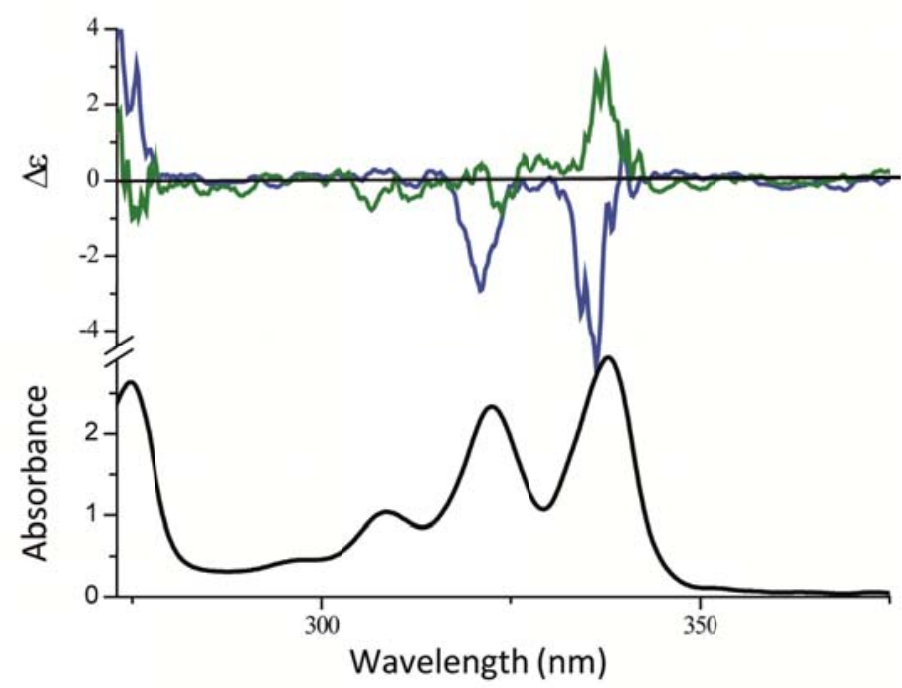

Fig. 6 Top: Circular dichroism spectra in NMP for aggregates GQD-E(R)/pyrene (blue) and GQD-E(S)/pyrene (green). Bottom: UV-vis spectrum of pyrene in NMP. 
The GQD-E(S)/pyrene aggregates showed in their CD spectrum a positive Cotton effect in the absorptions at 320-350 nm, whereas a negative Cotton effect is observed for the GQD-E(R)/pyrene aggregates in the same spectral range. Even when the obtained spectra are not specular images due to the most probable different degree of functionalization and organization in the CGQDs, the CD spectra evidenced the transfer of chirality from the CGQDs to the aggregate formed with pyrene and the divergent dichroic response of the nanoaggregates as a function of the $(R)$ or $(S)$ configuration of the chiral moieties introduced in the GQDs structure.

In summary, the covalent functionalization of GQDs with enantiomerically pure simple organic molecules results in the formation of the first CGQDs. The structural properties of the obtained chiral namomaterials have been investigated considering thermogravimetric analysis (TGA), X-ray diffraction (XRD) and different spectroscopic (NMR, FTIR, Raman, UV-Vis, fluorescence) and microscopic (TEM, AFM) techniques. As a result of the covalent functionalization, we proof the concept that GQDs could become chiral and that this property can be transferred to a supramolecular structure built with pyrene molecules, where the CGQDs/pyrene ensembles show a characteristic chiroptical response depending on the configuration of the organic ligands introduced. Based on this simple approach, is it possible to envision the construction of more sophisticated chiral carbon-based GQDs supramolecular organizations where chirality could play an important role for practical applications.

Financial support from the European Research Council (ERC-320441-Chiralcarbon), the UCM (GR3/14-910781), the Ministerio de Economía y Competitividad (MINECO) of Spain (Projects CTQ2014-52045-R and CTQ2015-71936REDT) and the CAM (PHOTOCARBON project S2013/MIT-2841) is acknowledged. N. M. thanks the Alexander von Humboldt Foundation.

\section{Notes and references}

1 J. L. Delgado, M. A. Herranz and N. Martín, J. Mater. Chem., 2008, 18, 1417.

2 H. W. Kroto, J. R. Heath, S. C. O’Brien, R. F. Curl and R. E. Smalley, Nature, 1985, 318, 162.

3 (a) S. lijima, Nature, 1991, 354, 56; (b) S. lijima and T. Ichihashi, Nature, 1993, 363, 603; (c) D. S. Bethune, C. H. Klang, M S. De Vries, G. Gorman, R. Savoy, J. Vasquez and R. Beyers, Nature, 1993, 363, 605.

4 K. S. Novoselov, A. K. Geim, S. V. Morosov, D. Jiang, Y. Zhang, S. V. Dubonos, I. V. Grigorieva and A. A. Firsov, Science, 2004, 306, 666.

5 (a) F. Langa and J. F. Nierengarten, Fullerenes Principles and Applications, RSC Publishers, Cambridge, 2012; (b) D. M. Guldi and N. Martín, Carbon Nanotubes and Related Structures, Wiley-VCH, Weinheim, 2010; (c) C. N. R. Rao and A. K. Sood, Graphene: Synthesis, Properties, and Phenomena, Wiley-VCH, Weinheim, 2013; (d) J. L. Delgado, S. Filippone, F. Giacalone, M. A. Herranz, B. Illescas, E. M. Pérez and N. Martín Top. Curr. Chem., 2014, 350, 1.

6 A. C. Ferrari, F. Bonaccorso, V. Fal'ko, K. S. Novoselov, S. Roche, P. Bøggild, S. Borini, F. H. L. Koppens, V. Palermo, N. Pugno, J. A. Garrido, R. Sordan, A. Bianco, L. Ballerini, M. Prato, E. Lidorikis, J. Kivioja, C. Marinelli, T. Ryhänen, A. Morpurgo, J. N. Coleman, V. Nicolosi, L. Colombo, A. Fert, M. García-Hernández, A. Bachtold, G. F. Schneider, F. Guinea, C. Dekker, M. Barbone, Z. Sun, C. Galiotis, A. N. Grigorenko, G. Konstantatos, A. Kis, M. Katsnelson, L. Vandersypen, A. Loiseau, V. Morandi, D. Neumaier, E. Treossi, V. Pellegrini, M. Polini, A. Tredicucci, G. M. Williams, B. H. Hong, J.-H. Ahn, J. M. Kim, H. Zirath, B. J. van Wees, H. van der Zant, L. Occhipinti, A. Di Matteo, I. A. Kinloch, T. Seyller, E. Quesnel, X. Feng, K. Teo, N. Rupesinghe, P. Hakonen, S. R. T. Neil, Q. Tannock, T. Löfwander and J. Kinaret, Nanoscale, 2015, 7, 4598.

7 (a) H. Li, Z. Kang, Y. Liu and S.-T. Lee, J. Mater. Chem., 2012, 22, 24230; (b) L. Li, G. Wu, G. Yang, J. Peng, J. Zhao and J.-J. Zhu, Nanoscale, 2013, 5, 4015.

8 Y. Wang, J. Xu, Y. Wang and H. Chen, Chem. Soc. Rev., 2013, 42, 2930.

9 M. P. Moloney, J. Govan, A. Loudon, M. Mukhina and Y. K. Gun'ko, Nature Protocols, 2015, 10, 558.

10 (a) S. Filippone, E. E. Maroto, A Martín-Domenech, M. Suarez and N. Martín, Nat. Chem., 2009, 1, 578; (b) E. E. Maroto, M. Izquierdo, S. Reboredo, J. Marco-Martínez, S. Filippone and N. Martín, Acc. Chem. Res., 2014, 47, 2660; (c) R. M. Girón, S. Reboredo, J. Marco-Martínez, S. Filippone and N. Martín, Faraday Discuss., 2014, $173,311$.

11 (a) F. Wang, K. Matsuda, A. F. M. M. Rahman, X. Peng, T. Kimura and N. Komatsu, J. Am. Chem. Soc., 2010, 132, 10876; (b) Y. Tsarfati, V. Strauss, S. Kuhri, E. Krieg, H. Weissman, E. Shimoni, J. Baram, D. M. Guldi and B. Rybtchinski, J. Am. Chem. Soc., 2015, 137, 7429.

12 R. Sekiya, Y. Uemura, H. Murakami and T. Haino, Angew. Chem. Int. Ed., 2014, 53, 5619.

13 F. Jiang, D. Chen, R. Li, Y. Wang, G. Zhang, S. Li, J. Zheng, N. Huang, Y. Gu, C. Wang and C. Shu, Nanoscale, 2013, 5, 1137.

14 L. Lin and S. Zhang, Chem. Commun., 2012, 48, 10177.

15 M. Zhang, L. Bai, W. Shang, W. Xie, H. Ma, Y. Fu, D. Fang, H. Sun, L. Fan, M. Han, C. Liu and S. Yang, J. Mater. Chem., 2012, 22, 7461.

16 (a) R. J. Chen, Y. Zhang, D. Wang and H. Dai, J. Am. Chem. Soc., 2001, 123, 3838; (b) C. B. KC, G. N. Lim and F. D'Souza, Angew. Chem. Int. Ed., 2015, 127, 5177; (c) E. M. Pérez and N. Martín, Chem. Soc. Rev., $2015,44,6425$. 


\section{Table of contents entry}

Graphene quantum dots covalently modified with enantiomerically pure $R / S$ units provide chiral graphene quantum dots that, upon assembly with pyrene molecules, show a strong chiroptical response.

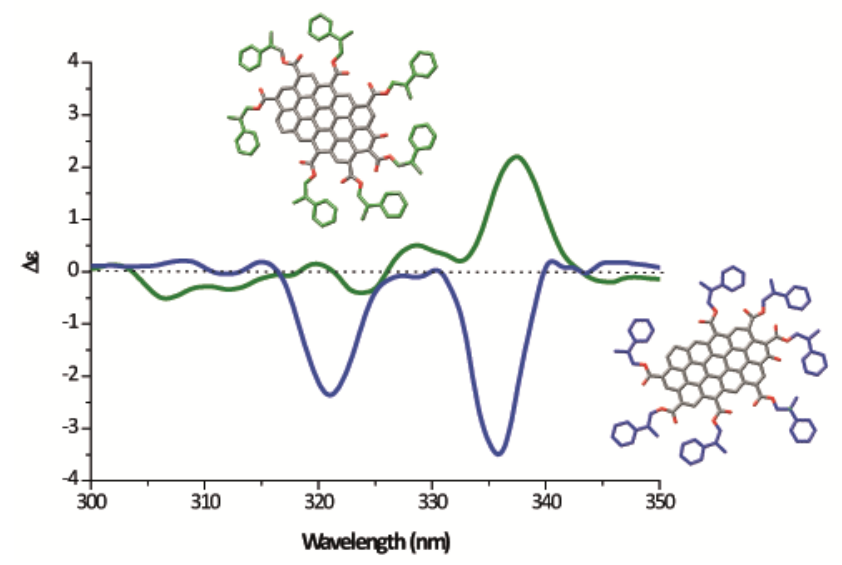

